\section{8 \\ SUSTENERE}

Publishing Corporation

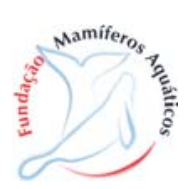

\section{(1)}

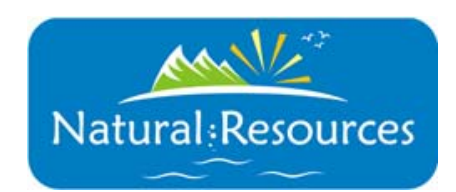

Journals Homepage:

www.sustenere.co/journals

\title{
INGESTÃO DE LIXO POR SAPO (Rhinella sp.)
}

\section{RESUMO}

Diariamente, milhões de toneladas de lixo são lançadas no ambiente, colocando em risco o equilíbrio da natureza. $O$ despejo de detritos no ambiente é considerado como uma das principais ameaças à vida silvestre em todo o mundo, estando relacionada como potencial fator de impacto para muitas espécies de aves e tartarugas marinhas e mamíferos aquáticos, os quais vem sofrendo com a poluição e ingestão acidental. O objetivo deste trabalho é relatar a ingestão de lixo plástico por um sapo do gênero Rhinella encontrado morto em bom estado de conservação na capital de Sergipe, Brasil. Durante a necropsia, observou-se que o animal apresentava pele íntegra e bom estado nutricional. Os órgãos da cavidade celomática apresentavam-se em sua topografia normal e, com exceção do estômago, sem alterações. O compartimento gástrico do anfíbio apresentava-se dilatado e, durante a palpação, notou-se a presença de conteúdo gástrico de aspecto folhoso. Após a sua abertura, foi identificada a presença de corpo estranho - material plástico correspondente a embalagens de balas. Até o momento, não há relatos de ingestão de lixo plástico por anfíbios no Brasil. A obstrução do trato digestivo pode desencadear um processo inflamatório, resultando na perda de apetite, caquexia, debilidade acentuada, podendo chegar ao óbito. Para que o problema do lixo no ambiente seja reduzido, é necessária a realização de campanhas educacionais voltadas para a orientação e educação da população sobre o impacto do lixo no equilíbrio ambiental.

PALAVRAS-CHAVES: Plástico; Sapo; Rhinella sp.

\section{GARBAGE INGESTION BY BEAKED TOAD (Rhinella sp.)}

\section{ABSTRACT}

Every day millions tons of garbage are released into the environment endangering the nature balance. The disposal of debris in the environment is considered one of the major threats to wildlife worldwide related as a potential impact for many species of marine birds, sea turtles and acquatic mammals which have been suffering with pollution and accidental ingestion. The aim of this study was to report the ingestion of plastic waste by a frog from the gender Rhinella, found dead in good condition in Aracaju, Sergipe, Brazil. The necropsy showed that the animal had intact skin and good nutrition. The coelomic cavity organs had normal topography and morphology, except for the stomach. The Rhinella gastric compartment was enlarged and, on palpation, the presence of gastric contents with leafy aspect was noted. After its opening, it was possible to observe the presence of a foreign body - a plastic material corresponding to candy packaging. Until now, there are no reports of plastic waste ingestion by amphibians in Brazil. The digestive tract obstruction may trigger an inflammatory process, resulting in loss of appetite, cachexia and severe weakness, leading to death. In order to reduce waste in the environment, educational campaigns are necessary, guiding and educating people about the impact of garbage on the environmental balance.

KEYWORDS: Plastic, Anfibiam, Rhinella sp.

Natural Resources, Aquidabã, v.4, n.1, Set, Out, Nov, Dez 2013, Jan, Fev, Mar, Abr, Mai, Jun, Jul, Ago 2014.

ISSN 2237-9290

\section{SECTION: Articles}

TOPIC: Efeitos Antropogênicos

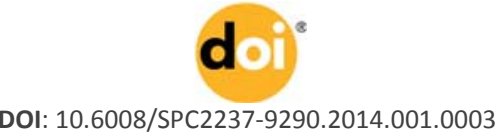

Fernanda Meneses Rodrigues
Pio Décimo, Brasil http://lattes.cnpq.br/2065276721525240 fmr09@hotmail.com

Ubatã Corrêa Pereira

Universidade Federal de Sergipe, Brasil http://lattes.cnpq.br/9223989142009404 ubata cp@hotmail.com

\section{Ana Carolina Trompieri} Silveira Pereira

Universidade Federal de Sergipe, Brasil http://lattes.cnpq.br/0829757313622548 actrompieri@yahoo.com.br

Juliana Plácido Guimarães

Universidade Santa Cecilia, Brasil http://lattes.cnpq.br/9781044537066765 juvet@usp.br

Received: 05/08/2013

Approved: 29/09/2014 Reviewed anonymously in the process of blind peer.

\section{Referencing this:}

RODRIGUES, F. M.; PEREIRA, U. C.; PEREIRA, A. C. T. S.; GUIMARÃES, J. P.. Ingestão de lixo por sapo (Rhinella sp.). Natural Resources, Aquidabã, v.4, n.1, p.24-28, 2014. DOI: http://dx.doi.org/10.6008/SPC2237$\underline{9290.2014 .001 .0003}$ 


\section{INTRODUÇÃO}

O crescimento populacional, a expansão territorial urbana, a ampliação do sistema de produção de resíduos e o consumo industrial contribuem para o crescimento de impactos ambientais negativos (MENDES et al., 2004; MUCELIN et al., 2008). Segundo Rodrigues (1998), um dos mais graves problemas ambientais da humanidade são os resíduos sólidos. Diariamente, milhões de toneladas de lixo são lançadas no ambiente, colocando em risco o equilíbrio da natureza. A prática de depositar resíduos ao ar livre teve início nas civilizações antigas, em que o método consistia em depositar os restos da atividade humana em ruas, rios, córregos e terrenos vazios (MENDES et al., 2004; GOUVEIA, 1999).

Com o aumento populacional a quantidade de lixo produzido vem crescendo significativamente, sendo esses muitas vezes depositados de forma inadequada no ambiente causando poluição que consiste em qualquer alteração das propriedades físicas, químicas ou biológicas do meio ambiente (MENDES et al., 2004), ocasionando problemas como o assoreamento de rios, entupimento de bueiros, destruição de áreas verdes, mau-cheiro, proliferação de moscas, baratas e ratos (GOUVEIA, 1999).

Segundo SILVANO et al. (2005), a principal ameaça à conservação dos anfíbios é a destruição de seus habitats, como consequência do desmatamento, avanço da fronteira agrícola, mineração, queimadas, desenvolvimento da infraestrutura, urbanização, doenças infecciosas, mudanças climáticas, espécies invasoras ou comércio de animais silvestres. O impacto do lixo no ambiente natural é documentado como uma das principais ameaças à vida destes animais (BLAUSTEIN et al., 2002; SILVA et al., 2009), pois envolve a poluição das águas e contaminação por pesticidas (YOUNG et al., 2001; 2004).

Sapos do gênero Rhinella são comuns na fauna brasileira e se alimentam de pequenos vertebrados e invertebrados. Esses anfíbios vivem em diferentes ambientes, incluindo áreas de praia, florestas, formação vegetal e ambientes urbanos (SARRAZIN et al., 2002). Eles se desenvolvem no meio aquático e terrestre, o que Ihes confere uma dupla vulnerabilidade. Assim, qualquer perturbação na água ou na terra pode afetá-los, o que os torna bons indicadores da saúde ambiental (FREITAS et al., 2004).

O presente trabalho tem como objetivo relatar a presença de lixo no estômago de um sapo do gênero Rhinella.

\section{RELATO}

Um sapo do gênero Rhinella (Figura 1A) foi encontrado morto, em bom estado de conservação e encaminhado para o laboratório de Patologia Animal do Hospital Veterinário Dr. Vicente Borelli da Faculdade Pio Décimo, Aracaju(SE) - Brasil, para realização da necropsia. 
Ao exame externo do animal notou-se pele íntegra e bom estado nutricional. Posteriormente, foi realizada a abertura da cavidade celomática, onde observou-se que os órgãos se apresentavam em sua topografia normal (Figura 1B).

Durante análise, observou-se que o estômago se apresentava dilatado e após a abertura do compartimento gástrico (Figura $1 \mathrm{C}$ ), constatou-se a presença de conteúdo estomacal e corpo estranho - material plástico correspondente a embalagens de balas (Figura 1D).

Possivelmente, o Rhinella relatado neste trabalho ingeriu o corpo estranho por não o distinguir de uma presa. Por conseguinte, o fato pode ter provocado obstrução do trato digestivo, podendo ter ocasionado o óbito do animal.
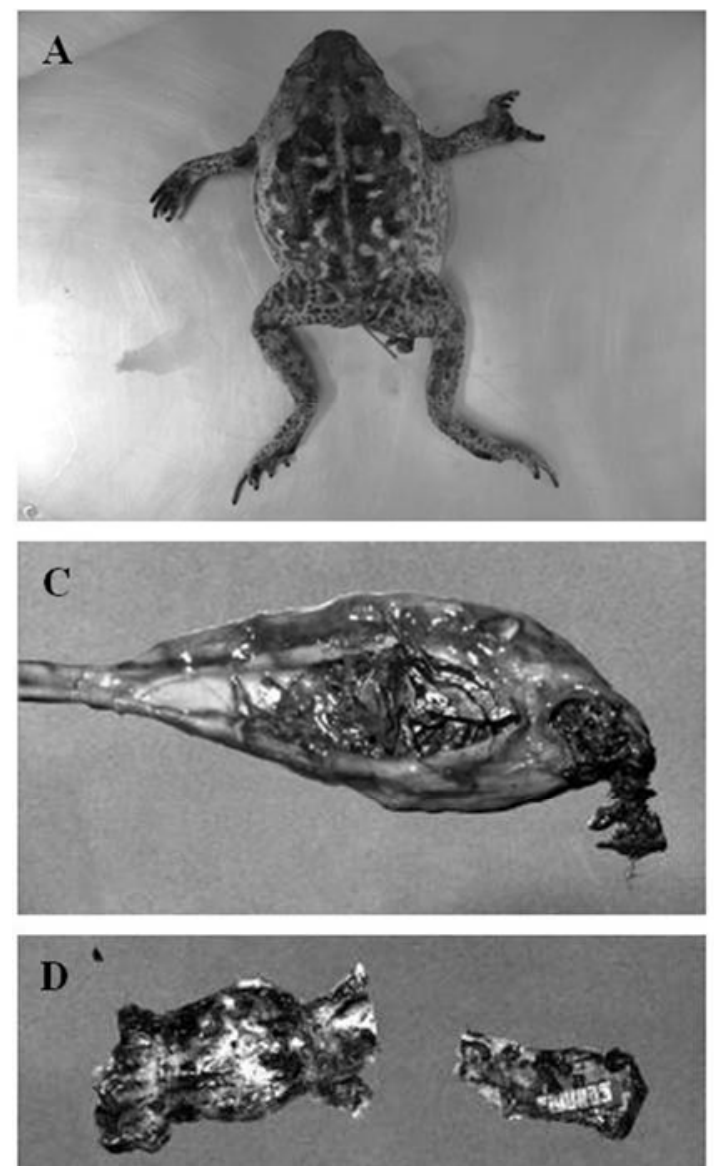

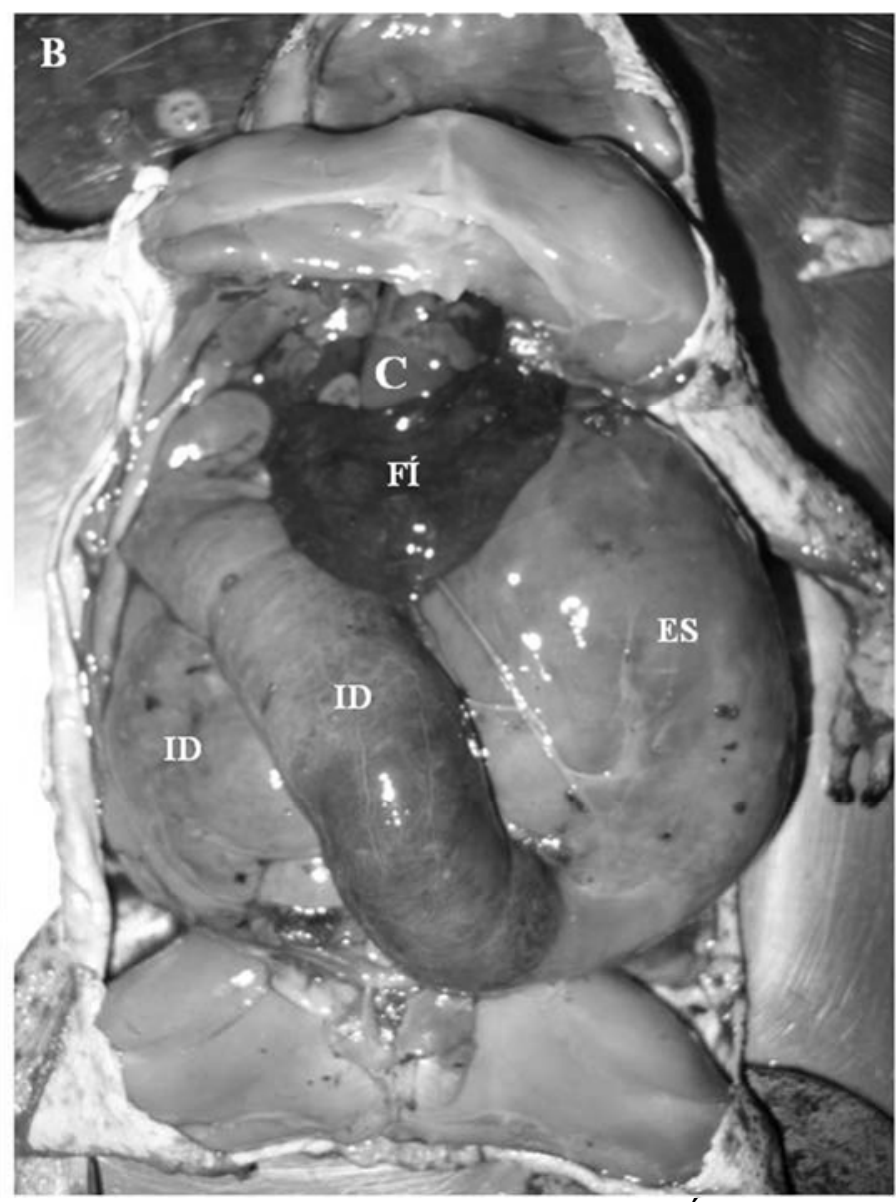

Figura 1: (A) Sapo do gênero Rhinella; (B) Vista geral dos órgãos internos; (C) coração; (Fí) fígado; (ES) estômago; (ID) intestino delgado; (C) Estômago de sapo do gênero Rhinella com presença de lixo; (D) Resíduos plásticos (embalagem de bala) encontrados no estômago.

\section{DISCUSSÃO}

Segundo dados do IBGE (2004), de todo o lixo produzido no Brasil, apenas $2 \%$ é coletado seletivamente e somente $6 \%$ das residências são atendidas por serviços de coleta seletiva em $8,2 \%$ dos municípios brasileiros, demonstrando o alto grau de deficiência na coleta pública de lixo, principalmente nas regiões norte e nordeste, e a falta de conscientização ambiental da população em geral (SILVA et al., 2009). 
Estudos indicam que o lixo afeta várias espécies animais em todo o mundo. Crustáceos, peixes, quelônios, aves e mamíferos marinhos, por exemplo, podem se ferir ou morrer por se prender em cintas de lixo plástico (como selos de garrafas ou partes de latas de cerveja) ou por ingerir todo tipo de objeto, mais comumente sacos plásticos e plástico rígido (LAIST et al., 1999).

Em algumas espécies encontra-se alta incidência de lixo no trato digestivo. Carcaças de aves marinhas, por exemplo, vem sendo encontradas no sul do Brasil com uma grande quantidade de lixo em seus compartimentos gástricos (MÄDER et al., 2010). No litoral sul da região metropolitana de Recife-PE, também se observou presença de lixo no aparelho digestivo de tartaruga marinha (MELO et al., 2010). Segundo Silva et al. (2009), uma sacola plástica presente no canal digestivo do peixe-boi amazônico pode ter provocado a morte do animal.

Entretanto, no ambiente terrestre, é sabido que o lixo atrai grande diversidade de insetos e animais que estão à procura de alimento. Além disso, a presença desses animais (como mosquito, mosca, barata, cobra, rato e sapo) reflete poluição e doenças (GOMES et al., 2010).

A interação entre o lixo no meio ambiente e os anfíbios não foi registrada no Brasil, principalmente no que se refere à ingestão.

\section{CONCLUSÕES}

Os sapos possuem importância ecológica no equilíbrio do ecossistema, controlando a população de insetos e servindo como fonte de alimento para outros animais. Salienta-se a importância de cooperação entre a população, indústria, prefeitura, órgãos reguladores e organizações da sociedade civil para efetivamente enfrentar o assunto do lixo no ambiente. $\mathrm{Na}$ maioria dos casos, entretanto, a solução resume-se a atitudes individuais, já que, em última instância é o indivíduo que toma a decisão de jogar o lixo na rua, no rio ou no cesto (SILVA et al., 2009).

A educação ambiental promove e estimula o valor da conservação dos animais silvestres no meio ambiente e o reconhecimento do homem como parte integrante da natureza, garantindo o bem estar dos animais e o equilíbrio do meio ambiente (SILVA et al., 2009).

\section{REFERÊNCIAS}

BLAUSTEIN, R. A.; KIESERCKER, J. M.. Complexity in conservation: lessons from the global decline of amphibian populations. Ecology Letters, v.5, n.4, p.597-608, 2002.

FREITAS, M. A.; SILVA, T. F. S.. Anfíbios na Bahia: um guia de identificação. Camaçari: Politeno, 2004.

GOMES, R. S.; TERRA, R.. O lixo na comunidade de Buraco Fundo, Gargaú, São Francisco do Itabapoana: um olhar sobre o manguezal. In: CIRCUITO DE INICIAÇÃO CIENTÍFICA DO CEFET, 4. Anais. Campos dos Goytacazes: IFF, 2010.

GOUVEIA, N.. Saúde e meio ambiente nas cidades: o desafio da saúde ambiental. Saúde e sociedade, v.8, n.1, p.49-61, 1999. 
LAIST, D. W.; COE, J. M.; O'HARA, K. J.. Marine debris pollution. In: TWISS JUNIOR., J. R.; REEVES, R. R.. Conservation and management of marine mammals. Washington: Smithsonian Institution Press, 1999.

MÄDER, A.; COSTA, A. S.; CASA JUNIOR, G. E.; SANDER, M.. Ingestão de lixo marinho por procelariformes arribados nas praias do Rio Grande do Sul. In: CONGRESSO BRASILEIRO DE OCEANOGRAFIA, 3. Anais. Rio Grande do Sul: AOCEANO, 2010.

MELO, C. M. F.; SANTOS, R. M. B.; AMORA, T. D.; OLIVEIRA, R. A. S.. Estudo do impacto fisiológico do lixo na tartaruga verde através da análise do aparelho digestivo. In: CONGRESSO BRASILEIRO DE OCEANOGRAFIA 3. Anais. Rio Grande do Sul: AOCEANO, 2010.

MENDES, I. L.; JAMES, O. T.. Geografia geral e do Brasil: estudos para a compreensão do espaço. São Paulo: FTD, 2004.

MUCELIN, C. A.; BELLINI, M.. Lixo e impactos ambientais perceptíveis no ecossistema urbano. Sociedade \& Natureza, Uberlândia, v.10, n.1, p.111-124, 2008.

RODRIGUES, A. M.. Produção e consumo do e no espaço: problemática ambiental urbana. São Paulo: Hucitec, 1998.

SARRAZIN, L. A. A.; COELHO, W.C.S.; YUKI, N.. Dieta de Bufo marinus (Linnaeus, 1758) de duas áreas de formação secundária da Amazônia Central (Amphibia: Anura). In: ENCONTRO ANUAL DE INICIAÇÃO CIENTÍFICA, 11. Anais. Maringá: Universidade Estadual de Maringá, 2002.

SILVA, A. B.; MARMONTEL, M.. Ingestão de lixo plástico como provável causa mortis de peixe-boi amazônico. Uakari, v.5, n.1, p.105-112, 2009.

SILVANO, D. L.; SEGALLA, M. V.. Conservação de anfíbios no Brasil. Megadiversidade, v.1, n.1, p.79-86, 2005.

YOUNG, B.; LIPS, K. R.; REASER, J. K.; IBANES, R.; SALAS, A. W.; CEDENO, J. R.; COLOMA, L. A.; RON, S.; MARCA, E. L; MEYER, J. R.; MUNOZ, A.; BOLANOS, F.; CHAVES, G.; ROMO, D.. Population declines and priorities for amphibian conservation in Latin America. Conservation Biology, v.15, p.12131223, 2001.

YOUNG, B. E.; STUART, S. N.; CHANSON, J. S.; COX, N. A.; BOUCHER, T. M.. Disappearing jewels: the status of New World amphibians. Virginia: NatureServe, 2004. 